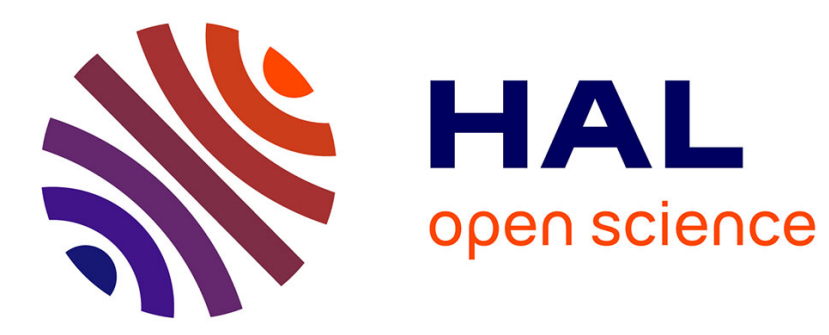

\title{
Multicontact Interaction Force Sensing From Whole-Body Motion Capture
}

\author{
Tu-Hoa Pham, Stéphane Caron, Abderrahmane Kheddar
}

\section{To cite this version:}

Tu-Hoa Pham, Stéphane Caron, Abderrahmane Kheddar. Multicontact Interaction Force Sensing From Whole-Body Motion Capture. IEEE Transactions on Industrial Informatics, 2018, 14 (6), pp.2343-2352. 10.1109/TII.2017.2760912 . hal-01610928

\section{HAL Id: hal-01610928 \\ https://hal.science/hal-01610928}

Submitted on 5 Oct 2017

HAL is a multi-disciplinary open access archive for the deposit and dissemination of scientific research documents, whether they are published or not. The documents may come from teaching and research institutions in France or abroad, or from public or private research centers.
L'archive ouverte pluridisciplinaire HAL, est destinée au dépôt et à la diffusion de documents scientifiques de niveau recherche, publiés ou non, émanant des établissements d'enseignement et de recherche français ou étrangers, des laboratoires publics ou privés. 


\title{
Multi-Contact Interaction Force Sensing from Whole-Body Motion Capture
}

\author{
Tu-Hoa Pham, Stéphane Caron, and Abderrahmane Kheddar, Senior Member, IEEE
}

\begin{abstract}
We present a novel technique that unobtrusively estimates forces exerted by human participants in multi-contact interaction with rigid environments. Our method uses motion capture only, thus circumventing the need to setup cumbersome force transducers at all potential contacts between the human body and the environment. This problem is particularly challenging, as the knowledge of a given motion only characterizes the resultant force, which can generally be caused by an infinity of force distributions over individual contacts. We collect and release a large-scale dataset on how humans instinctively regulate interaction forces on diverse multi-contact tasks and motions. The force estimation framework we propose leverages physicsbased optimization and neural networks to reconstruct force distributions that are physically realistic and compatible with real interaction force patterns. We show the effectiveness of our approach on various locomotion and multi-contact scenarios.
\end{abstract}

Index Terms-Force sensing from motion capture, neural networks, physics-based optimization, whole-body, multi-contact.

\section{INTRODUCTION}

$\mathbf{H}$ UMAN motions result from skilled control of the physical interactions with the environment through contacts. Thus, haptic perception is a fundamental theme towards action understanding and control. The monitoring of contact forces is already widely used in various fields such as robot learning from demonstration and control [1], [2], physics-based animation [3], [4], and visual tracking [5], [6]. Measurement of contact forces is usually achieved by mounting force transducers at pre-fixed contact locations, making it a costly, cumbersome and intrusive process that is difficult to use in daily settings. Mounting force transducers on the persons obstructs their natural motion and is not sustainable for daily use. In contrast, the accurate monitoring of interaction forces from motion capture alone, which can readily be achieved using consumer-grade cameras [7], [8], would enable a wide range of applications in personal robotics, human-computer interaction, and rehabilitation [9] as a new unobtrusive biosensor for the healthcare Internet of Things [10].

However, this problem is very difficult due to the indeterminacy of force distributions in multi-contact. Indeed, while the knowledge of external and internal forces uniquely determines the resulting kinematics for a given articulated system, even a perfectly known motion does not suffice to fully characterize

Manuscript received November 21, 2016; revised May 12, 2017; accepted October 2, 2017.

T.-H. Pham, S. Caron and A. Kheddar are with the Interactive Digital Humans group of CNRS-University of Montpellier LIRMM, UMR5506, Montpellier, France. T.-H. Pham and A. Kheddar are also with the CNRSAIST Joint Robotics Laboratory, UMI3218/RL, Tsukuba, Japan, the underlying forces in multi-contact. Instead, the resultant force can be distributed in infinitely many different ways on a given set of contacts. Illustrating this indeterminacy, consider a human participant standing still with both feet on the ground. Even in the elementary case of a static biped stance, the participant can exert tangential forces that compensate each other out, e.g., by pushing their feet apart. While substantial work was dedicated to the problem of force indeterminacy during gait, general contact configurations (e.g., involving hands) have been comparatively less studied in the literature (Section II).

We address the force distribution problem in multi-contact by combining the benefits of machine learning techniques and physics-based optimization to capture the variability in the way humans naturally regulate interaction forces while ensuring their physical compatibility with the observed motion.

- We formulate an optimization problem allowing the estimation of physically valid forces either from motion observations alone or from a reference signal (Section III).

- We construct a novel dataset on human whole-body kinodynamics containing $2.4 \mathrm{~h}$ of synchronized force and motion measurements under diverse configurations of tasks, participants and contacts (Section IV).

- We propose two neural network architectures allowing the prediction of contact force distributions from motion observations as well as their interactive correction by physics-based optimization (Section V)

- We validate our approach with ground-truth force measurements on various multi-contact scenarios and assess the respective contributions of physics-based optimization and neural networks (Section VI)

Finally, we discuss the limitations, applications and future extensions of our work (Section VII). Besides a significantly extended dataset, our current work enhances the earlier approach of [11] with: an improved formulation of the optimization problem accounting for motion measurement uncertainties, the consideration of individual contact normals in the learning features enabling more fine-grained predictions by neural network models, as well as algorithmic descriptions and extensive validation experiments that have not been presented before. To foster the research on this new topic and encourage alternative implementations, we make the whole-body kinodynamics dataset and algorithms publicly available ${ }^{1}$.

${ }^{1}$ https://github.com/jrl-umi3218/WholeBodyKinodynamics. 


\section{RELATED WORK}

Research on human-computer interaction has resulted in multiple techniques for whole-body motion capture from markerless visual observations [7], [8], magnetic trackers [12] or wearable inertial sensors [13], [14]. Force sensors were notably used in conjunction with inertial sensors and vision to improve the motion reconstruction in [3], [4]. Instead of physical force sensors, numerical models were used to compute physically plausible distributions supporting visual observations in hand-object tracking [5], [6]. The problem of estimating the real forces applied on the environment was tackled in the case of deformable objects [15] and conversely by considering the human body elastic [16]. In the inspiring work of [17], ground reaction forces were computed with a spring-based contact model to estimate internal joint torques during locomotion. General contact configurations are commonly addressed in simulation and robotics using constrained optimization [18], which alone may not result in the forces humans instinctively apply, as illustrated in Section III-C.

Inverse optimization approaches in kinesiology research address the force distribution indeterminacy by modeling the objective function(s) supposedly optimized by the central nervous system [19]. However, such approches are difficult due to the redundancy of the human body and the difficulty to observe physiological parameters without invasive surgery [20]. The variability of inverse dynamics solutions with different body segment inertial parameter (BSIP) models was notably discussed in [21], [22]. Towards this issue, [23] introduced an optimization framework for the online estimation of robot and human BSIPs from motion and force-torque measurements. An alternative approach for BSIP reconstruction was proposed in [24], along with a data-driven approach to estimate contact forces from motion tracking between the feet and the ground.

Recent successes for the control of robot arms [25], [26] or general articulated characters [27] using neural networks illustrated their ability to account for complex model uncertainties. Neural networks were also used to resolve force indeterminacy cases during gait [28] and manipulation [29]. To account for temporal continuity, recurrent neural networks (RNN) [30] with long short-term memory (LSTM) [31] neurons were used in [32], [33], still for manipulation. Whole-body interactions were first addressed using an RNN in combination with a second-order program (SOCP) [34] for physics-based optimization in [11]. Our current study generalizes this idea to more complex multi-contact scenarios, supported by an extended dataset that is significantly more diverse in terms of contact configurations, tasks and participants.

\section{Whole-Body Contact Force Optimization}

\section{A. Equations of Motion and Friction Constraints}

We consider an articulated system of rigid bodies subject to $N_{\boldsymbol{\tau}}$ internal joint torques $\boldsymbol{\tau}=\left(\tau_{1}^{(i)}, \ldots, \tau_{N_{\boldsymbol{\tau}}}^{(i)}\right)^{T}$ and $N_{\mathbf{F}}$ external wrenches $\mathbf{F}_{k}=\left(\boldsymbol{\tau}_{k}, \mathbf{f}_{k}\right)^{T}$, with $\boldsymbol{\tau}_{k}$ and $\mathbf{f}_{k}$ the respective external torque and force at contact $k$, expressed in the global frame. With the position and orientation of a chosen base link, the number of degrees of freedom is $N_{D o F}=N_{\tau}+6$.
We denote by $\mathbf{q}, \dot{\mathbf{q}}, \ddot{\mathbf{q}}$ the respective generalized coordinates, velocity and acceleration of the articulated system. The wholebody equations of motion can be expressed as:

$$
\mathbf{H}(\mathbf{q}) \ddot{\mathbf{q}}+\mathbf{C}(\mathbf{q}, \dot{\mathbf{q}})=\left[\begin{array}{c}
\mathbf{0}_{6} \\
\boldsymbol{\tau}
\end{array}\right]+\sum_{k=1}^{N_{\mathbf{F}}} \mathbf{J}_{k}^{T} \mathbf{F}_{k},
$$

with:

- $\mathbf{H}(\mathbf{q})$ the $N_{D o F} \times N_{D o F}$ mass matrix,

- $\mathbf{C}(\mathbf{q}, \dot{\mathbf{q}})$ the $N_{D o F} \times 1$ bias vector of the Coriolis, centrifugal forces and gravity terms,

- $\mathbf{J}_{k}$ the $N_{D o F} \times 6$ the $k^{\text {th }}$ contact Jacobian matrix,

- $\mathbf{0}_{6}$ the $6 \times 1$ internal wrench directly applied at the root of the kinematic tree in case of linkage with the environment (zero for the case of the floating base).

We assume the parameters of the dynamic model to be known [23], [24]. For each contact $k$, we denote by $\mathbf{z}_{k}$ the (uniquely defined) normal vector oriented from the environment to the body, and by $\mathbf{x}_{k}$ and $\mathbf{y}_{k}$ two orthogonal vectors in the tangential plane. We thus obtain a local decomposition for each external wrench $\mathbf{F}_{k}$ in the contact frame $\mathcal{C}_{k}=$ $\left(\mathbf{x}_{k}, \mathbf{y}_{k}, \mathbf{z}_{k}\right)$ :

$$
\begin{aligned}
& { }^{\mathcal{C}_{k}} \mathbf{F}_{k}=\left(\tau_{k}^{\mathbf{x}}, \tau_{k}^{\mathbf{y}}, \tau_{k}^{\mathbf{z}}, f_{k}^{\mathbf{x}}, f_{k}^{\mathbf{y}}, f_{k}^{\mathbf{z}}\right)^{T}, \\
& \text { with }\left\{\begin{array}{c}
\boldsymbol{\tau}_{k}=\tau_{k}^{\mathbf{x}} \mathbf{x}_{k}+\tau_{k}^{\mathbf{y}} \mathbf{y}_{k}+\tau_{k}^{\mathbf{z}} \mathbf{z}_{k} \\
\mathbf{f}_{k}=f_{k}^{\mathbf{x}} \mathbf{x}_{k}+f_{k}^{\mathbf{y}} \mathbf{y}_{k}+f_{k}^{\mathbf{z}} \mathbf{z}_{k}
\end{array} .\right.
\end{aligned}
$$

Having chosen $\mathbf{z}_{k}$ oriented towards the body, each normal force component is such that:

$$
f_{k}^{\mathbf{z}} \geq 0
$$

With $\mu_{k}$ the friction coefficient at contact $k$, the tangential force is constrained by the normal component as follows:

$$
\left\|f_{k}^{\mathbf{x}} \mathbf{x}_{k}+f_{k}^{\mathbf{y}} \mathbf{y}_{k}\right\|_{2} \leq \mu_{k} f_{k}^{\mathbf{z}} .
$$

Contact torque constraints are usually obtained by discretizing the contact surface into individual contact points subject to 3D forces only. Closed-form formulae were derived for rectangular support areas in [35]. We observed in our experiments that such constraints could be violated due to motion tracking uncertainties and omitted them in this study.

\section{B. Physics-Based Optimization}

In this section, we discuss the extraction of physically plausible force distributions compatible with a given motion, characterized by generalized coordinates $\mathbf{q}$, velocities $\dot{\mathbf{q}}$ and accelerations $\ddot{\mathbf{q}}$. Such force distributions can be obtained as solutions of a second-order cone program (SOCP) of the form:

$$
\begin{aligned}
& \min \mathcal{C}(\mathbf{x})=\frac{1}{2} \mathbf{x}^{T} \mathbf{P} \mathbf{x}+\mathbf{r}^{T} \mathbf{x} \\
& \text { s.t. }\left\{\begin{aligned}
\left\|\mathbf{A}_{j} \mathbf{x}+\mathbf{b}_{j}\right\|_{2} & \leq \mathbf{c}_{j}^{T} \mathbf{x}+\mathbf{d}_{j}, \quad j=1, \ldots, m \\
\mathbf{E x} & \leq \mathbf{f} \\
\mathbf{G x} & =\mathbf{h},
\end{aligned}\right.
\end{aligned}
$$

with $\mathrm{x}$ a vector of $N_{\mathbf{x}}=6+N_{\boldsymbol{\tau}}+6 N_{\mathbf{F}}$ force variables:

$$
\mathbf{x}=\left(\mathbf{F}^{\mathrm{MEW}}, \boldsymbol{\tau},\left({ }^{\mathcal{C}_{k}} \mathbf{F}_{k}\right)_{k=1, N_{\mathbf{F}}}\right)^{T} \text {. }
$$


Here, $\mathbf{F}^{\mathrm{MEW}}$ represents a measurement error wrench (MEW) applied to the floating base of the kinematic tree. This wrench is $\mathbf{0}_{6}$ in the ideal case of perfect measurements and dynamic model. However, trying to enforce the strict constraint $\mathbf{F}^{\mathrm{MEW}}=\mathbf{0}_{6}$ on noisy measurements and with an approximative dynamic model results in unfeasible SOCP problems. To allow for uncertainties, we relax this constraint and rather make the solver enforce it at best (i.e., minimizing $\left\|\mathbf{F}^{\mathrm{MEW}}\right\|$ ), as detailed thereafter.

Inequality constraints. In Eq. (5), linear inequality matrices $\mathbf{E}, \mathbf{f}$ and cone inequality matrices $\mathbf{A}, \mathbf{b}_{j}, \mathbf{c}_{j}, \mathbf{d}_{j}$ can directly be computed from Eqs. (3) and (4), respectively.

Equality constraints. We consider the whole-body equations of motion. Given an instance of $(\mathbf{q}, \dot{\mathbf{q}}, \ddot{\mathbf{q}})$, the term $\mathbf{h}$ in Eq. (5) corresponds directly to the left-hand side of Eq (1):

$$
\mathbf{h}=\mathbf{H}(\mathbf{q}) \ddot{\mathbf{q}}+\mathbf{C}(\mathbf{q}, \dot{\mathbf{q}}) .
$$

$\mathbf{h}$ is a vector of $N_{D o F}$ elements. The matrix $\mathbf{G}$ in $\mathrm{Eq}$ (5) is here of size $N_{D o F} \times N_{\mathbf{x}}$ and can be decomposed using selection matrices $\mathbf{G}_{\boldsymbol{\tau}}$ and $\left(\mathbf{G}_{\mathbf{F}_{k}}\right)_{k=1, N_{\mathbf{F}}}$ such that:

$$
\mathbf{G}_{\boldsymbol{\tau}} \mathbf{x}=\left[\begin{array}{c}
\mathbf{F}^{\mathrm{MEW}} \\
\boldsymbol{\tau}
\end{array}\right] \text { and } \quad \mathbf{G}_{\mathbf{F}_{k}} \mathbf{x}=\mathbf{F}_{k}
$$

Note that each $\mathbf{G}_{\mathbf{F}_{k}}$ must incorporate the rotation matrix between the contact frame $\mathcal{C}_{k}$ and the world frame. We obtain:

$$
\mathbf{G}=\mathbf{G}_{\boldsymbol{\tau}}+\sum_{k=1}^{N_{\mathbf{F}}} \mathbf{J}_{k}^{T} \mathbf{G}_{\mathbf{F}_{k}}
$$

Cost function. Having incorporated the previous constraints in the SOCP, physically plausible force distributions can be computed by minimizing a chosen cost function depending only on the optimization variables, e.g., a weighted sum of the squared $L^{2}$ norms of the optimization variables:

$$
\mathcal{C}_{\alpha, \beta, \gamma}(\mathbf{x})=\alpha\left\|\mathbf{F}^{\mathrm{MEW}}\right\|^{2}+\beta\|\boldsymbol{\tau}\|^{2}+\gamma \sum_{k=1}^{N_{\mathbf{F}}}\left\|\mathbf{F}_{k}\right\|^{2} .
$$

In practice, it is preferrable to set $\alpha$ greater than $\beta$ and $\gamma$ so that $\mathbf{F}^{\mathrm{MEW}}$ is only used when the observed motion is otherwise unfeasible. The two other parameters $\beta$ and $\gamma$ can be tuned to minimize either internal joint torques or applied contact wrenches. Alternatively, when target values $\widetilde{\mathbf{F}}_{k}$ for the contact wrenches are available (e.g., from force-torque sensors), it is possible to extract force distributions in their vicinity that are also guaranteed to be physically plausible, by minimizing the discrepancy to the optimized wrenches in the SOCP cost function [36]:

$$
\mathcal{C}_{\alpha, \beta, \gamma}^{\widetilde{\mathbf{F}}_{k}}(\mathbf{x})=\alpha\left\|\mathbf{F}^{\mathrm{MEW}}\right\|^{2}+\beta\|\boldsymbol{\tau}\|^{2}+\gamma \sum_{k=1}^{N_{\mathbf{F}}}\left\|\mathbf{F}_{k}-\widetilde{\mathbf{F}}_{k}\right\|^{2}
$$

Contact forces and internal joint torques occurring during gait are typically in the order of $100 \mathrm{~N}$ and $1 \mathrm{~N}$ m respectively [21]. In our experiments, we chose $\alpha=10^{2}, \beta=10^{-2}$ and $\gamma=1$ so that $\mathbf{F}^{\mathrm{MEW}}$ only compensates unfeasible raw motion measurements and internal joint torques can vary as needed to prioritize matching optimized and target contact wrenches.

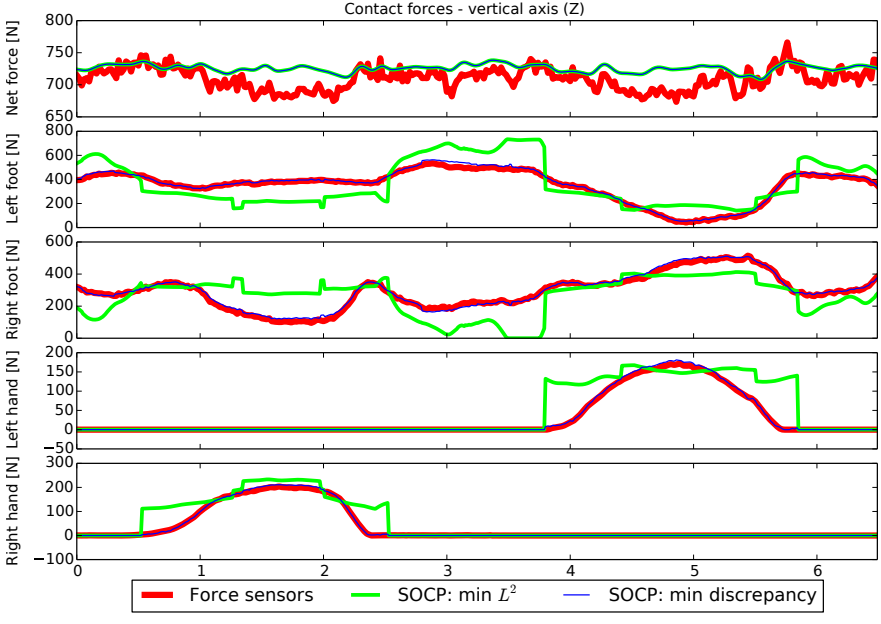

Fig. 1. Force sensor noise and uncertainties in the resultant force (top plot) can be corrected using physics-based optimization. In multi-contact, directly minimizing the norm of the individual forces (green) results in forces that are physically plausible but significantly differ from real measurements (red). By minimizing the discrepancy to the latter (blue), we reconstruct forces that are both physically plausible and in agreement with natural force distributions.

\section{Motivating Example: Triple Contact Indeterminacy}

We illustrate the crucial role played by the SOCP cost function. We consider a participant standing still next to a table and taking support on it using the right hand, then the left. We represent the vertical component of the measured forces in Fig. 1. In addition, we compute force distributions of minimal $L^{2}$ norm using Eq. (10) and minimizing the discrepancy to the sensor measurements using Eq. (11).

With the participant standing still, the equations of motion dictate that the net contact forces (top plot) should mostly oppose the participant's weight. However, individual force sensor uncertainties result in rather noisy force estimates. In contrast, all SOCP variants accurately reconstruct the net force directly from the measured kinematics. Using the cost function of Eq. (10) results in forces that are physically plausible but may greatly differ from actual measurements. Using the cost function of Eq. (11) enables the reconstruction of force distributions that are both physically plausible and in the vicinity of target forces when available. The aim of our work is to circumvent the need for force sensors. Thus, in the following, we train recurrent neural networks to predict such target force distributions directly from motion observations.

\section{Whole-Body Kinodynamics Dataset}

\section{A. Experimental Setup}

We depict our complete acquisition system in Fig. 2. Whole-body motion. We track the whole-body motion using the Xsens MVN Awinda inertial motion capture system [13], comprised of 17 inertial measurement units (IMU) worn and strapped at specified body landmarks on the participant's body. The motion capture system is battery-powered and wireless, transmitting accelerometer, gyroscope and magnetometer measurements to the computer at $100 \mathrm{~Hz}$. The motion of the human body, modeled as a 23-segment skeleton, is then readily provided in the form of the $6-\mathrm{DoF}$ pose, velocity 


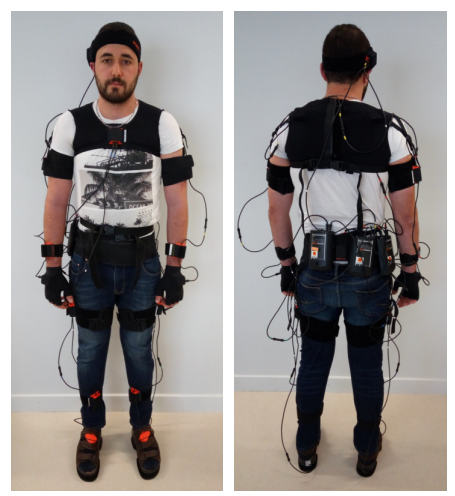

(a) Inertial motion capture system.

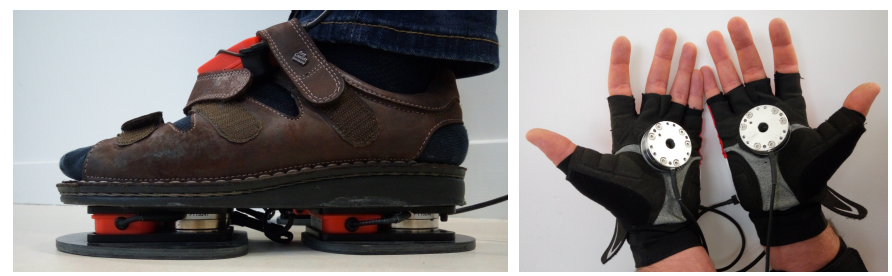

(c) Shoes and gloves instrumented with force-torque sensors.

Fig. 2. Acquisition system for whole-body kinematics and contact forces.

and acceleration of each segment. We enable the dynamics analysis of Section III by converting these quantities into generalized coordinates, velocities and accelerations $(\mathbf{q}, \dot{\mathbf{q}}, \ddot{\mathbf{q}})$, with a kinematic tree composed of 23 segments linked by 22 spherical joints and rooted at the participant's pelvis. Measuring the participant's body measurements and weight, we compute the BSIPs using the anthropomorphic tables of [37]. Inertial motion capture systems by themselves do not provide absolute positioning and are prone to drift compared to marker-based tracking methods (e.g., Vicon). We are working towards attenuating this problem. Still, the choice of this motion capture system was motivated by strong occlusions that are inherent to whole-body interactions with the environment and hinder vision-based motion capture systems (e.g., Vicon). In contrast, the inertial motion capture system allows us to explore various interaction scenarios in uncontrolled and cluttered environments, e.g., when crouching under a table. In the future, the system could even be employed outdoors, or used in combination with a limited number of visual sensors to solve the issue of drift and absolute positioning.

Contact forces. We measure the contact forces exerted by the participant onto the environment both at the feet and at the hands. Contact forces at the feet are monitored using instrumented shoes (Xsens ForceShoe). Each shoe is equipped with two force-torque sensors and two IMUs, providing contact forces measured individually at the heel and toes, and transmitted to the computer via Bluetooth at $50 \mathrm{~Hz}$. We monitor contact forces exerted at the hands using two additional force-torque sensors (ATI Mini-45) attached to gloves worn by the participant during interaction experiments. The forcetorque sensors are wired to dedicated acquisition cards on the computer and measurements are also recorded at $50 \mathrm{~Hz}$. Both ForceShoe and ATI sensor signals are linearly interpolated to $100 \mathrm{~Hz}$, matching the motion capture sampling rate. In comparison to static force plates commonly used in gait analysis, wearable force sensors can be less accurate. Still, a major advantage of our lightweight setup is that it enables the efficient and continuous acquisition of contact forces on arbitrary contact configurations, highly dynamic motions, and relatively unrestricted movement areas. In contrast, using static force plates considerably reduce the range of possible tasks, contacts and motions.

\section{B. Newton-Euler Equations and Signal Synchronization}

Each type of sensor used in this work (i.e., motion capture suit, force-sensing shoes, ATI Mini-45 sensors) is individually monitored using a dedicated acquisition program. Therefore, raw measurements need to be temporally synchronized with each other before further analysis. This step is performed using the Newton-Euler equations taken at the center of mass $\mathbf{G}$ of the whole-body articulated system. For each body segment $s$ of the 23-element set $\mathcal{S}$, we denote by $m_{s}$ its mass and $\mathbf{G}_{s}$ its center of mass. In the global frame, we denote by $\mathbf{v}_{s}$ the linear velocity of $\mathbf{G}_{s}$ and $\mathbf{R}_{s}$ its orientation matrix. In the segment frame, we denote by $\boldsymbol{\omega}_{s}$ and $\mathbf{I}_{s}$ its local angular velocity and inertia tensor, respectively. With $m$ the total mass of the articulated system and $\mathbf{G}$ its centroid, the linear momentum $\mathcal{P}$ and angular momentum $\mathcal{L}_{\mathbf{G}}$ at $\mathbf{G}$ are defined by:

$$
\left\{\begin{aligned}
\mathcal{P} & =\sum_{s \in \mathcal{S}} m_{s} \mathbf{v}_{s} \\
\mathcal{L}_{\mathbf{G}} & =\sum_{s \in \mathcal{S}} m_{s} \overrightarrow{\mathbf{G G}_{s}} \times \mathbf{v}_{s}+\mathbf{R}_{s} \mathbf{I}_{s} \boldsymbol{\omega}_{s}
\end{aligned}\right.
$$

With $\dot{\mathcal{L}}_{\mathrm{G}}$ and $\dot{\mathcal{P}}$ the time derivatives of the angular and linear momenta, respectively, $\mathbf{g}$ the gravity vector and ${ }^{\mathbf{G}} \mathbf{F}_{k}$ the contact wrench at contact $k$ transformed to $\mathbf{G}$, the NewtonEuler equations for centroidal dynamics state that:

$$
\left[\begin{array}{c}
\dot{\mathcal{L}}_{\mathbf{G}} \\
\dot{\mathcal{P}}
\end{array}\right]=\left[\begin{array}{c}
\mathbf{0} \\
m \mathbf{g}
\end{array}\right]+\sum_{k=1}^{N_{\mathbf{F}}} \mathbf{G}_{\mathbf{F}_{k}} .
$$

We gather gravity, linear and angular momenta as a centroidal wrench $\mathbf{w}^{\mathbf{G}}$ due to contact forces, taken at $\mathbf{G}$ [38]:

$$
{ }_{\mathbf{w}}{ }^{\mathbf{G}}=\left[\begin{array}{c}
\dot{\mathcal{L}}_{\mathbf{G}} \\
\dot{\mathcal{P}}-m \mathbf{g}
\end{array}\right]
$$

With $\mathbf{P}_{k}$ the location of contact $k$, Eq. (13) becomes:

$$
{ }_{\mathbf{w}} \mathbf{G}=\sum_{k=1}^{N_{\mathbf{F}}}\left[\begin{array}{l}
\boldsymbol{\tau}_{k}+\overrightarrow{\mathbf{G P}_{k}} \times \mathbf{f}_{k} \\
\mathbf{f}_{k}
\end{array}\right] .
$$

${ }_{\mathbf{w}^{\mathbf{G}}}$ is a purely kinematic term that can be directly computed from the whole-body pose and its derivatives using Eq. (14), but also from the contact forces using Eq. (15). Thus, synchronizing motion capture and force measurements amounts to synchronizing $\mathbf{w}^{\mathbf{G}}$ estimates from kinematics and forces. For this purpose, we start each experiment by having the participant walk a few seconds, then take support on a table with the left and right hand, alternatively. To synchronize kinematic and ForceShoe signals, we plot the components of their respective estimates for $\mathbf{w}_{\text {kin }}^{\mathbf{G}}$ and $\mathbf{w}_{\text {shoe }}^{\mathbf{G}}$ during the 
walking phase and select by hand a constant time shift to match the two signals at best. We then compute the residual wrench $\mathbf{w}_{\text {res }}^{\mathbf{G}}=\mathbf{w}_{\text {kin }}^{\mathbf{G}}-\mathbf{w}_{\text {shoe }}^{\mathbf{G}}$. When the participants leans on a table with one hand, $\mathbf{w}_{\text {res }}^{\mathbf{G}}$ should be equal to the wrench $\mathbf{w}_{\text {hand }}^{\mathbf{G}}$ measured by the corresponding force-torque sensor. Again, we find a constant time shift to match $\mathbf{w}_{\text {hand }}^{\mathbf{G}}$ and $\mathbf{w}_{\text {res }}^{\mathbf{G}}$ at best, thus synchronizing hand sensors with kinematic-ForceShoe signals.

Following the temporal synchronization, we perform the following signal processing. All measurements are subject to noise, e.g., from the sensors themselves or due to interferences in the transmission (both wired and wireless). We attenuate it by smoothing all signals with a Gaussian filter of kernel $\sigma=0.05 \mathrm{~s}$. In addition, a slow-varying bias can appear in the force-torque measurements with repeated stress and battery drain. We estimate this bias through time by averaging the signals that persist when the sensors are not in contact with the environment, which should only be caused by the inertia of the moving parts attached to the sensing surface (e.g., force shoe external sole). Since the inertial motion capture system does not provide absolute positioning, we could not reliably identify the occurrence of contacts with the environment based solely on the whole-body motion observations. Therefore, we identified them by direct thresholding on the force sensor measurements. Still, this material limitation does not affect the generality of our approach and can be fully circumvented with additional visual observations (see also [39] for the retrieval of contact points without environment knowledge). Finally, we correct the remaining force sensor uncertainties by combining their measurements with the motion capture data using the SOCP approach illustrated in Section III-C. In the following, we call ground truth the SOCP-corrected sensor measurements (relative to the dynamic model).

\section{Experiments}

The importance of collecting ground-truth measurements on how humans naturally distribute contact forces not only during locomotion, but across a variety of multi-contact configurations was established in [11]. Thus, in this work, we purposefully explore a wide range of motion dynamics as well as diverse contact configurations that exhibit strong force distribution indeterminacy. The following tasks were chosen from daily activities to cover a spectrum of three features: number of contacts involved, orientation of hand contacts (when applicable) and effort required to perform the motion:

- Walking, i.e. with always at least one foot on the ground (1 contact, low effort). Straight and curved paths were considered separately;

- Running, i.e. with at most one foot on the ground (1 contact, medium effort);

- Hopping on one foot, e.g., forward or in place (1 contact, high effort);

- Balancing the upper body while keeping both feet static, e.g., leg stretching or performing arm motions ( 2 contacts, low effort);

- Jumping using both feet, e.g., forward or to the side (2 contacts, high effort);
- Taking support on a table with one hand, e.g., to reach for an object further on the table (3 contacts, horizontal hand contact, low effort);

- Crouch and stand by taking support with one hand on a table, e.g., to reach for an object under the table (3 contacts, horizontal hand contact, high effort);

- Leaning against a wall with one hand (3 contacts, vertical hand contact, low effort);

- Leaning on a wall with one hand and reach forward, e.g., to look around a corner or grab an object (3 contacts, vertical hand contact, high effort);

- Taking support on a table with both hands (4 contacts, horizontal hand contacts, low to high effort);

- Leaning on a wall with both hands, e.g., to stretch or push a heavy object (4 contacts, vertical hand contacts, low to high effort).

Contact is a complementarity condition involving the dual geometric and force spaces. The first two features we used to categorize our tasks (namely number and orientations of contacts) ensure coverage of the geometric part of the condition, while the last one (perceived effort) aims for coverage of the force space. Considering the two variants (straight and curved) of walking experiments separately, we thus construct a repertoire of twelve motion types, six of them involving contacts between the feet and the ground only and the six others involving both feet and hands. We illustrate this dataset in Table I.

Six volunteers, three males and three females, took part in our study. Their weights (between $45.0 \mathrm{~kg}$ and $86.0 \mathrm{~kg}$, plus the $5.0 \mathrm{~kg}$ acquisition system), heights (between $1.57 \mathrm{~m}$ and $1.92 \mathrm{~m}$ ), and individual body segment lengths were measured to initialize the motion capture skeletal tracking model and BSIPs following the procedure described in Section IV-A. Before each experiment, all sensors (i.e., inertial motion capture system, force-sensing shoes and glove-mounted force sensors) were calibrated and reset following the manufacturers' recommended acquisition procedure to reduce the effects of measurement drift and hysteresis. We divided the 12 motion types of Table I into two sequences of 6 motions. Each sequence consisted in 3 tasks involving the hands and 3 tasks involving only the feet, executed in alternation for one minute each. Participants were given time between consecutive tasks to put on, or take off instrumented gloves, so locomotion tasks were not constrained by unnecessary force sensor wires. In total, each task was executed twice by each participant. For one particpant, we observed force measurement errors of abnormal magnitude on the right-hand sensor and discarded the corresponding recordings from the dataset. For another participant, the motion capture system was disconnected during a hopping task. Overall, our new dataset on human whole-body kinodynamics in multi-contact totals $2.4 \mathrm{~h}$ of synchronized motion and force measurements, classified into 12 task primitives.

\section{Capturing Human Force Distribution Patterns}

\section{A. Learning Features}

Let $\mathbf{K}$ denote a set of (input) whole-body kinematic features, and $\mathbf{D}$ a set of (output) contact force features. The 
TABLE I

Interaction Configurations From the Whole-Body Kinodynamics Dataset.

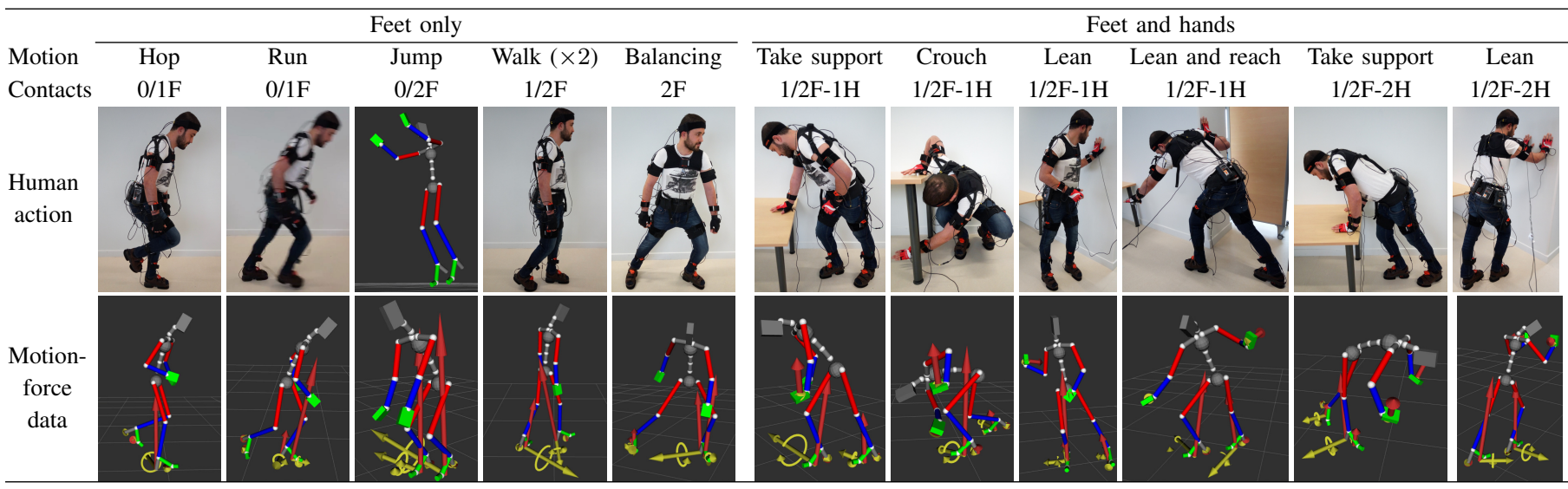

desired contact force estimation mapping $\mathcal{F}$ is of the form:

$$
\mathbf{D}=\mathcal{F}(\mathbf{K})
$$

We model this mapping $\mathcal{F}$ using a neural network trained on our whole-body kinodynamics dataset. The dynamic features $\mathbf{D}$ simply correspond to the set of contact wrenches $\mathbf{F}_{k}$ we seek to estimate. A straightforward approach to construct the set of kinematic features $\mathbf{K}$ could be to take all the remaining parameters appearing in the whole-body equations of motion of Eq. (1), e.g., the mass $\mathbf{H}$ and bias $\mathbf{C}$ matrices, joint accelerations $\ddot{\mathbf{q}}$, and Jacobian matrices $\mathbf{J}_{k}$ representing the contact configuration. However, doing so would result in a particularly large number of parameters that can make the neural network training process difficult. We instead propose to construct a selection of high-level kinematic features based on the Newton-Euler equations of Eq. (13), which extract the gist of locomotory dynamics. In particular, from the formulation of Eq. (15), we take as first input features the centroidal wrench $\mathbf{w}^{\mathrm{G}}$, which can be computed from kinematics only with Eq. (14), and the contact positions relative to the center of mass, $\overrightarrow{\mathbf{G P}_{k}}$. Since these quantities are expressed in the world frame, we account for translational and rotational invariances by transforming them to a reference frame $\mathcal{G}$ of origin $\mathbf{G}$ and fixed with respect to a chosen body segment (e.g., the pelvis). Walking straight to the North is thus locally equivalent to walking straight to the East. To facilitate the modeling of the mapping of Eq. (16) with a neural network, we construct $\mathrm{K}$ as a fixed-size input vector. We continuously monitor $N_{c}$ potential contacting body segments over time and encode their activity with parameters $\delta_{k, i}$ such that:

$$
\delta_{k, i}= \begin{cases}1 & \text { if contact } k \text { is active at time step } i \\ 0 & \text { otherwise }\end{cases}
$$

In our experiments, we considered the forces applied at the heels and toes separately in both the SOCP and the neural network model, so that $N_{c}=6$ including the hand palms. Finally, in addition to the contact locations, we consider their orientation through the contact normals $\mathbf{z}_{k}$. Denoting by ${ }^{\mathcal{G}}{ }_{\mathbf{w}}^{\mathbf{G}}$,

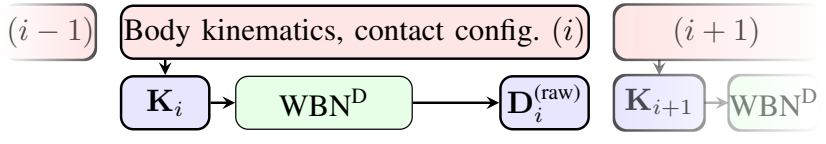

(a) Forces are direcly computed from the kinematics and contact configuration.

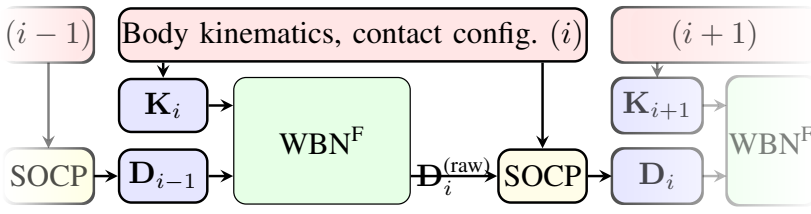

(b) Force predictions are corrected between consecutive time steps.

Fig. 3. Direct and feedback whole-body network architectures.

${ }^{\mathcal{G}} \mathbf{P}_{k},{ }^{\mathcal{G}} \mathbf{z}_{k}$ the respective coordinates of ${ }^{\mathbf{G}}, \mathbf{P}_{k}, \mathbf{z}_{k}$ in the frame $\mathcal{G}$, the complete input features at time step $i$ are:

$$
\mathbf{K}_{i}=\left({ }^{\mathcal{G}} \mathbf{w}_{i}^{\mathbf{G}},\left({ }^{\mathcal{G}} \mathbf{P}_{k, i}, \delta_{k, i}, \mathbf{z}_{k, i},\right)_{k=1, N_{c}}\right)^{T} .
$$

Similarly, the output features are the target wrenches in $\mathcal{G}$ :

$$
\mathbf{D}_{i}=\left(\left({ }^{\mathcal{G}} \mathbf{F}_{k, i}\right)_{k=1, N_{c}}\right)^{T} .
$$

\section{B. Neural Network Architecture}

We model the evolution of motion and force distributions as time series using RNNs with LSTM neurons in order to account for temporal continuity between consecutive samples. In this section, we propose two neural network architectures to be used in conjunction with physics-based optimization. The first architecture, $\mathrm{WBN}^{\mathrm{D}}$ (whole-body network, direct), directly maps the observed motion to the underlying forces:

$$
\mathbf{D}_{i}=\mathrm{WBN}^{\mathrm{D}}\left(\mathbf{K}_{i}\right) .
$$

Once trained, this network is used as follows. At each step, a new vector of kinematic features $\mathbf{K}_{i}$ is fed to $\mathrm{WBN}^{\mathrm{D}}$, yielding raw output features $\mathbf{D}_{i}^{\text {(raw) }}$. Since the RNN model does not enforce the equations of motion, the corresponding forces $\mathbf{F}_{k, i}^{\text {(raw) }}$ may not be readily compatible with the observed motion. We compute physically plausible forces $\mathbf{F}_{k, i}$ in their vicinity using the SOCP of Eq. (5) with the cost function of Eq. (11). 
TABLE II

Force ESTIMATION ERRoRs ON FUll TESTING Set ( $23 \mathrm{~min}$ )

\begin{tabular}{lcc}
\hline & \multicolumn{1}{c}{ Raw } & SOCP correction \\
\cline { 2 - 3 } Force sensors & $1.6 \%$ & ground truth \\
SOCP min. $L^{2}$ & N/A & $7.0 \%$ \\
$\mathrm{WBN}^{\mathrm{D}}$ & $8.3 \%$ & $6.4 \%$ \\
$\mathrm{WBN}^{\mathrm{F}}$ & $6.6 \%$ & $5.8 \%$ \\
\hline
\end{tabular}

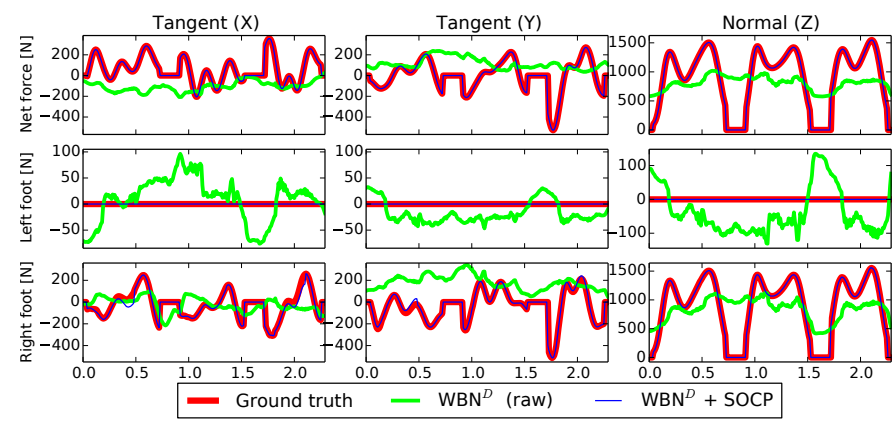

Fig. 4. SOCP correction on single contact motion (hop on the right foot).

Alternatively, we enable the interactive correction of RNN predictions by constructing a network $\mathrm{WBN}^{\mathrm{F}}$ (whole-body network, feedback) that takes as inputs both the current kinematics and the distribution at the previous time step:

$$
\mathbf{D}_{i}=\mathrm{WBN}^{\mathrm{F}}\left(\mathbf{K}_{i}, \mathbf{D}_{i-1}\right) .
$$

When using $\mathrm{WBN}^{\mathrm{F}}$ for prediction, we initialize $\mathbf{D}_{0}$ to the distribution of minimal $L^{2}$ norm following Eq. (10). At each time step, $\mathbf{K}_{i}$ and $\mathbf{D}_{i-1}$ are fed together to $\mathrm{WBN}^{\mathrm{F}}$, yielding raw predictions $\mathbf{D}_{i}^{\text {(raw) }}$ By SOCP correction, we reconstruct physically accurate forces $\mathbf{F}_{k, i}$ and extract the corresponding dynamic features $\mathbf{D}_{i}$, used for prediction at the next time step.

We depict the two proposed architectures in Fig. 3. Note that for $\mathrm{WBN}^{\mathrm{D}}$, raw predictions $\mathbf{D}_{i}^{\text {(raw) }}$ may be corrected independently from each other, enabling opportunities for parallel computing if desired. In contrast, the intertwined RNN-SOCP approach of $\mathrm{WBN}^{\mathrm{F}}$ imposes a sequential prediction process.

\section{RESULTS}

\section{A. Prediction-Correction Framework}

We implement the two proposed neural network architectures within the Torch7 framework [40] as two LSTM hidden layers of size 256 followed by a linear output layer of size $6 N_{c}$, the number of output features. We partition the wholebody kinodynamics dataset into three subsets of respective size $70 \%, 15 \%$ and $15 \%$ for training, validation and testing. We train the neural networks by minimizing a mean square error regression criterion using mini-batch stochastic gradient descent and dropout to avoid overfitting [41]. We estimated from the dataset that participants maintained each contact on average for $2.07 \mathrm{~s}$. We thus set the length of the training batches to $2.0 \mathrm{~s}$. The SOCP correction is implemented separately using the CVXOPT library for convex optimization [42]. We run the prediction process for each task of the testing set and compute the root mean square errors (RMSE) between reconstructed forces and ground truth distributions. We normalize the RMSEs with the range of the normal forces measured in the testing set, $f_{\max }^{\mathbf{z}}=1378 \mathrm{~N}$. For the sake of completeness, we also quantify the force sensor measurement uncertainties, the estimation errors for distributions computed by straightforward minimization of their $L^{2}$ norm, and prediction errors for the neural networks alone, without SOCP correction. We report the resulting normalized RMSE (NRMSE) in Table II.

Expectedly, the lowest estimation errors are attained using physical force sensors, that directly measure the applied contact wrenches. Still, this level of accuracy was obtained using costly, cumbersome force sensors. Table II yields three major outcomes. First, we confirm the previous observation that physics-based optimization alone does not suffice to address the issue of force indeterminacy in multi-contact, since the $L^{2}$ minimizing cost function provides the worst results of the 2 nd column. Second, even without SOCP correction (first column), the accuracy of $\mathrm{WBN}^{\mathrm{F}}$ exceeds that of $\mathrm{WBN}^{\mathrm{D}}$, and even that of the $L^{2}$-minimizing SOCP alone. Thus, RNNs can successfully capture interaction force patterns even without enforcing the equations of motion during training. In particular, the better performance of $\mathrm{WBN}^{\mathrm{F}}$ compared to $\mathrm{WBN}^{\mathrm{D}}$ shows that providing the RNN with past forces as inputs helps handle force indeterminacy, i.e., associating a given motion (unique $\mathbf{K}_{i}$ ) to multiple possible distributions (different $\mathbf{D}_{i}$ ). Third, combining RNN and SOCP yields the best results overall, improving the accuracy of $\mathrm{WBN}^{\mathrm{D}}$ and $\mathrm{WBN}^{\mathrm{F}}$ by $23 \%$ and $11 \%$ respectively, and that of the SOCP alone by $17 \%$.

\section{B. Accuracy in Multi-Contact Indeterminacy}

The effectiveness of the SOCP to correct inaccurate force predictions is particularly visible for the hopping sequence depicted in Fig. 4. Indeed, for this motion, the presence of only one foot on the ground at each instant makes it straightforward for the SOCP to enforce that the force exerted at the only contact is exactly causing the acceration of the centroid. We further investigate the respective contributions of RNN and SOCP by separating experiments with only feet or with both feet and hands. Since the former involves relatively large impulses (e.g., during jumping), we normalize the estimation errors of each category by the range of their respective measurements. We report the resulting NRMSEs in Table III. For both categories, combining RNN and SOCP yields significant improvements compared to either in isolation. Importantly, the NRMSEs of all three estimation methods are larger when also considering hand contacts, which illustrates the increased multi-contact indeterminacy.

Finally, we further decompose the tasks involving feet and hands and assess the estimation accuracy by body segment in Table IV. For all configurations, again, the SOCP greatly improves the accuracy of both neural network architectures. However, while for the feet, the three estimation methods yield comparable NRMSEs, the estimation errors of the SOCP alone on the hands (rightmost column) are now significantly larger than that of WBN variants. This result shows that recurrent neural networks are well suited to tackle the issue of force indeterminacy in multi-contact, for which physics-based optimization can serve as a valuable complement. 
TABLE III

ESTIMATION ERRORS BY CONTACT CONFIGURATION

\begin{tabular}{|c|c|c|c|c|}
\hline & \multicolumn{2}{|c|}{$\begin{array}{c}\text { Feet only }(13 \mathrm{~min}) \\
f_{\max }^{\mathbf{z}}=1378 \mathrm{~N}\end{array}$} & \multicolumn{2}{|c|}{$\begin{array}{c}\text { Feet }+ \text { hands }(10 \mathrm{~min}) \\
f_{\max }^{\mathbf{z}}=750 \mathrm{~N}\end{array}$} \\
\hline & Raw & SOCP & Raw & SOCP \\
\hline Force sensors & $2.1 \%$ & ground truth & $1.9 \%$ & ground truth \\
\hline $\mathrm{SOCP} \min . L^{2}$ & N/A & $8.7 \%$ & N/A & $9.6 \%$ \\
\hline $\mathrm{WBN}^{\mathrm{D}}$ & $9.9 \%$ & $7.6 \%$ & $12.2 \%$ & $9.4 \%$ \\
\hline $\mathrm{WBN}^{\mathrm{F}}$ & $7.5 \%$ & $6.6 \%$ & $10.3 \%$ & $9.3 \%$ \\
\hline
\end{tabular}

TABLE IV

ESTIMATION ERRORS By SEGMENT ON FEET + HAND TASKS (10 min)

\begin{tabular}{|c|c|c|c|c|}
\hline & \multicolumn{2}{|c|}{ Feet: $f_{\max }^{\mathbf{z}}=750 \mathrm{~N}$} & \multicolumn{2}{|c|}{ Hands: $f_{\max }^{\mathbf{z}}=177 \mathrm{~N}$} \\
\hline & Raw & SOCP & Raw & SOCP \\
\hline Force sensors & $2.0 \%$ & ground truth & $5.7 \%$ & ground truth \\
\hline $\mathrm{SOCP} \min . L^{2}$ & N/A & $10.8 \%$ & N/A & $21.9 \%$ \\
\hline $\mathrm{WBN}^{\mathrm{D}}$ & $14.2 \%$ & $11.0 \%$ & $14.4 \%$ & $10.5 \%$ \\
\hline $\mathrm{WBN}^{\mathrm{F}}$ & $12.0 \%$ & $10.8 \%$ & $13.2 \%$ & $12.9 \%$ \\
\hline
\end{tabular}

We depict sample force reconstruction results for two-, three- and four-contact motions in Fig. 5. In all cases, we confirm that the net force is reconstructed accurately by all methods, as expected. In the two-contact balancing scenario, we see that the $\mathrm{WBN}^{\mathrm{D}}$ network fails to capture weight shifts between feet ( $\mathrm{Z}$ component, rightmost column) and tends to predict uniform distributions, while the $\mathrm{WBN}^{\mathrm{F}}$ network tracks them suitably thanks to its ability to capture timedependent variations. With more contacts, time-dependency over pressure-distribution becomes less significant and both networks perform reasonably.

\section{DISCUSSION AND FUTURE WORK}

Our work establishes that the estimation of interaction forces, a problem that pertains to the human sense of touch, could be tackled through the lens of motion capture. The dual optimization and learning framework we propose extends the state of the art in capturing human force distribution patterns beyond gait analysis, to general multi-contact configurations used to interact with the environment. This important result makes it possible to completely circumvent costly, cumbersome and intrusive transducing technologies with any wholebody tracking system. Indeed, while we collected our (public) dataset using inertial motion capture, the RNN architectures we propose only rely on centroidal dynamics, making them agnostic with respect to the actual motion capture system employed. Meanwhile, an SOCP can be formulated for any whole-body kinematic model. As such, our framework is readily compatible with existing markerless visual tracking techniques, thus enabling novel interfaces for in-home, unobtrusive force monitoring for personal robotics or rehabilitation.

In its current implementation, our work has some limitations. First we consider the academic point-contact model, whereas in practice contacts are between surfaces, yielding additional complementarity conditions [35] that are, as we observed, difficult to take into account under motion-tracking

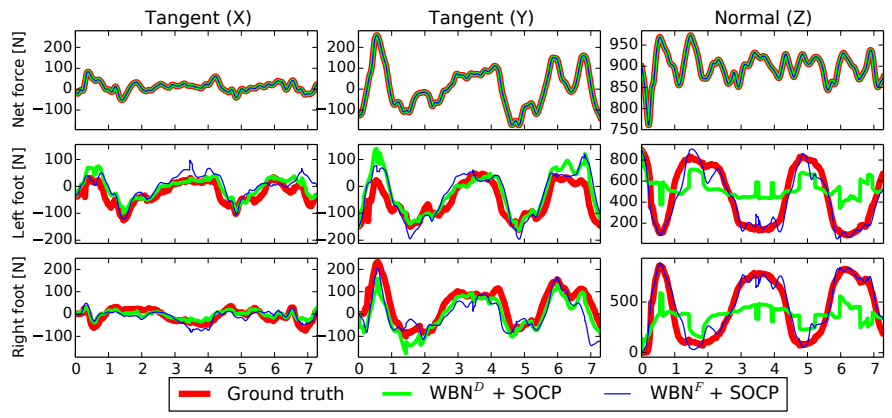

(a) Two contacts: upper-body balancing with static feet.

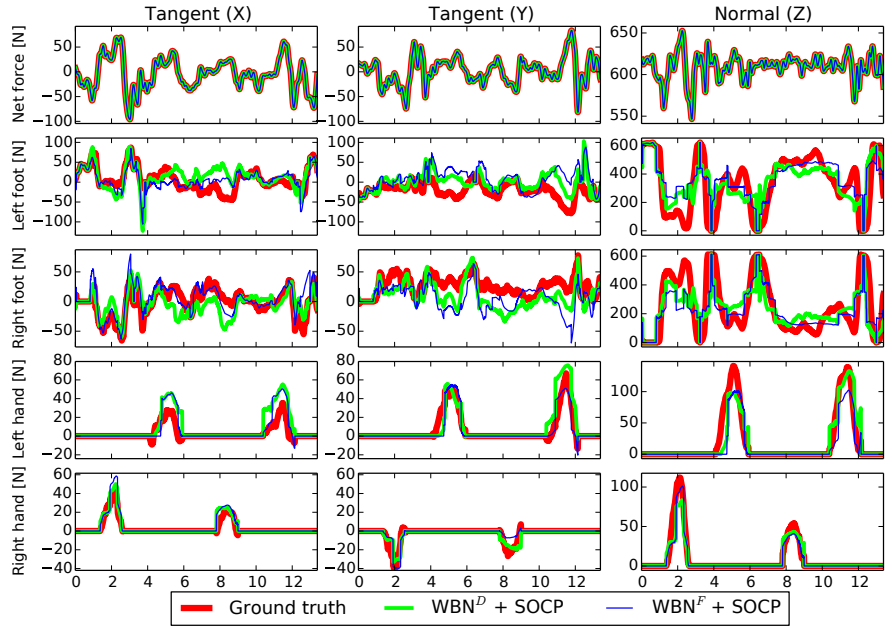

(b) Three contacts: taking support on a table with one hand (alternating).

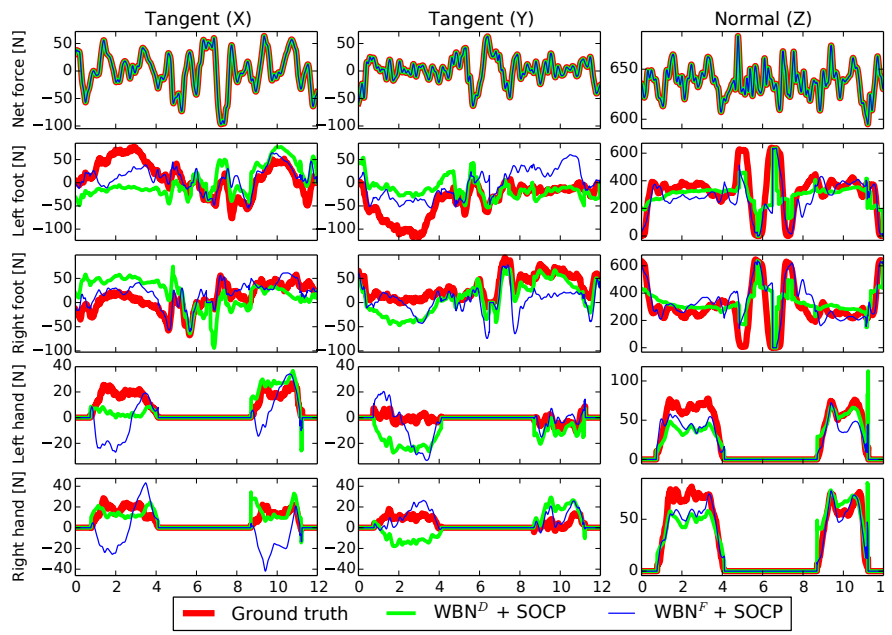

(c) Four contacts: leaning against a wall with two hands at the same time.

Fig. 5. Force profiles in various contact configurations. Net forces are measured in the world frame, while contact forces are reported in their respective (local) contact frames.

uncertainties. Contacts also include a certain amount of deformation that we did not model. Assessing the contact force by a portable force sensor also affects the natural motion behavior. Instead, one could distribute force sensing devices in the experimented environment, but at the cost of many more sensing units. Considering all body limbs for contact would be presently difficult, as wearable force sensing suits do not exist in the current state of the technology. We therefore chose to focus on foot and hand contacts, at the expense of other 
kinds of interaction such as shoulder or waist contacts (e.g. for seated motions).

To deal with these limitations and consider other features, our work can (and should) be extended to arbitrary contact configurations and motions. While a short-term solution could be to collect additional force and motion measurements (e.g., with force sensors at the knees and elbows), we anticipate that the increased level of instrumentation would strongly interfere with natural interaction behaviors, or even render some impossible (e.g., performing a cartwheel). Instead, our future work involves considering the distribution of contact forces as an inverse optimal control problem, i.e., finding optimization criteria privileging the forces measured in reality. Note that in the meantime, we ensured that the forces estimated by our framework would always be at least physically plausible (if not resembling human forces) by making the SOCP formulation independent of the acquired dataset. In the long term, we also plan to apply our framework to force-based robot learning from demonstration, on-line multi-contact motion retargeting and knowledge-based multi-contact planning and control [43].

\section{ACKNOWLEDGMENTS}

All participants gave informed consent prior to participation and the study was approved by the Ethics and Safety Committee of the University of Montpellier, France. This work was partially supported by the H2020 RIA COMANOID project (www.comanoid.eu), by JSPS Grant-in-Aid for Scientific Research (B) Number 16H02886 ("Cutting-edge multicontact behaviors) and by the Bpifrance project ROMEO 2 (www.projetromeo.com).

\section{REFERENCES}

[1] L. Rozo, P. Jiménez, and T. Carme, "A robot learning from demonstration framework to perform force-based manipulation tasks," Intelligent Service Robotics, vol. 6, no. 1, pp. 33-51, 2013.

[2] J. Englsberger, P. Kozlowski, and C. Ott, "Biologically inspired deadbeat controller for bipedal running in 3d," in IEEE/RSJ International Conference on Intelligent Robots and Systems, 2015, pp. 989-996.

[3] S. Ha, Y. Bai, and C. K. Liu, "Human motion reconstruction from force sensors," in ACM SIGGRAPH/Eurographics Symposium on Computer Animation, 2011, pp. 129-138.

[4] P. Zhang, K. Siu, J. Zhang, C. K. Liu, and J. Chai, "Leveraging depth cameras and wearable pressure sensors for full-body kinematics and dynamics capture," ACM Trans. on Graphics, vol. 33, no. 6, p. 221, 2014.

[5] N. Kyriazis and A. A. Argyros, "Physically plausible 3d scene tracking: The single actor hypothesis," in IEEE Conference on Computer Vision and Pattern Recognition, 2013, pp. 9-16.

[6] Y. Wang, J. Min, J. Zhang, Y. Liu, F. Xu, Q. Dai, and J. Chai, "Videobased hand manipulation capture through composite motion control," ACM Trans. on Graphics, vol. 32, no. 4, p. 43, 2013.

[7] C. Tran and M. M. Trivedi, "3-d posture and gesture recognition for interactivity in smart spaces," IEEE Trans. on Industrial Informatics, vol. 8, no. 1, pp. 178-187, 2012.

[8] D. Michel, K. Panagiotakis, and A. A. Argyros, "Tracking the articulated motion of the human body with two rgbd cameras," Machine Vision Applications, vol. 26, no. 1, pp. 41-54, 2015.

[9] A. González, M. Hayashibe, and P. Fraisse, "Subject-specific center of mass estimation for in-home rehabilitation-kinect-wii board vs. viconforce plate," in Converging Clinical and Engineering Research on Neurorehabilitation. Springer, 2013, pp. 705-709.

[10] G. Yang, L. Xie, M. Mäntysalo, X. Zhou, Z. Pang, L. Da Xu, S. KaoWalter, Q. Chen, and L.-R. Zheng, "A health-iot platform based on the integration of intelligent packaging, unobtrusive bio-sensor, and intelligent medicine box," IEEE Trans. on Industrial Informatics, vol. 10, no. 4, pp. 2180-2191, 2014.
[11] T.-H. Pham, A. Bufort, S. Caron, and A. Kheddar, "Whole-body contact force sensing from motion capture," in IEEE/SICE International Symposium on System Integration, 2016, pp. 58-63.

[12] S. J. Lee, Y. Motai, and H. Choi, "Tracking human motion with multichannel interacting multiple model," IEEE Trans. on Industrial Informatics, vol. 9, no. 3, pp. 1751-1763, 2013.

[13] D. Roetenberg, H. Luinge, and P. Slycke, "Xsens mvn: full 6dof human motion tracking using miniature inertial sensors," Xsens Motion Technologies BV, Tech. Rep, 2009.

[14] H. Ghasemzadeh and R. Jafari, "Physical movement monitoring using body sensor networks: A phonological approach to construct spatial decision trees," IEEE Trans. on Industrial Informatics, vol. 7, no. 1, pp. 66-77, 2011.

[15] M. Mohammadi, T. L. Baldi, S. Scheggi, and D. Prattichizzo, "Fingertip force estimation via inertial and magnetic sensors in deformable object manipulation," in IEEE Haptics Symposium, 2016, pp. 284-289.

[16] Y. Zhu, C. Jiang, Y. Zhao, D. Terzopoulos, and S.-C. Zhu, "Inferring forces and learning human utilities from videos," in IEEE Conference on Computer Vision and Pattern Recognition, 2016, pp. 3823-3833.

[17] M. A. Brubaker, L. Sigal, and D. J. Fleet, "Estimating Contact Dynamics," in IEEE International Conference on Computer Vision, 2009, pp. 2389-2396.

[18] S. Nakaoka, S. Hattori, F. Kanehiro, S. Kajita, and H. Hirukawa, "Constraint-based dynamics simulator for humanoid robots with shock absorbing mechanisms," in IEEE/RSJ International Conference on Intelligent Robots and Systems, 2007, pp. 3641-3647.

[19] V. M. Zatsiorsky, Kinetics of human motion. Human Kinetics, 2002.

[20] B. I. Prilutsky and V. M. Zatsiorsky, "Optimization-based models of muscle coordination," Exercise and Sport Sciences Reviews, vol. 30, no. 1, p. 32, 2002.

[21] G. Rao, D. Amarantini, E. Berton, and D. Favier, "Influence of body segments' parameters estimation models on inverse dynamics solutions during gait," Journal of Biomechanics, vol. 39, no. 8, pp. 1531-1536, 2006.

[22] A. Muller, C. Germain, C. Pontonnier, and G. Dumont, "A comparative study of 3 body segment inertial parameters scaling rules," Computer Methods in Biomechanics and Biomedical Engineering, vol. 18, no. sup1, pp. 2010-2011, 2015.

[23] J. Jovic, A. Escande, K. Ayusawa, E. Yoshida, A. Kheddar, and G. Venture, "Humanoid and human inertia parameter identification using hierarchical optimization," IEEE Trans. on Robotics, vol. 32, no. 3, pp. 726-735, 2016.

[24] X. Lv, J. Chai, and S. Xia, "Data-driven inverse dynamics for human motion," ACM Transactions on Graphics (SIGGRAPH Asia), vol. 35, no. 6, pp. 163:1-163:12, November 2016.

[25] C. Yang, Y. Jiang, Z. Li, W. He, and C.-Y. Su, "Neural control of bimanual robots with guaranteed global stability and motion precision," IEEE Trans. on Industrial Informatics, 2016.

[26] S. Levine, P. Pastor, A. Krizhevsky, J. Ibarz, and D. Quillen, "Learning hand-eye coordination for robotic grasping with deep learning and largescale data collection," International Journal of Robotics Research, 2016.

[27] I. Mordatch, K. Lowrey, G. Andrew, Z. Popovic, and E. V. Todorov, "Interactive control of diverse complex characters with neural networks," in Advances in Neural Information Processing Systems, 2015, pp. 31323140.

[28] S. E. Oh, A. Choi, and J. H. Mun, "Prediction of ground reaction forces during gait based on kinematics and a neural network model," Journal of biomechanics, vol. 46, no. 14, pp. 2372-2380, 2013.

[29] T.-H. Pham, A. Kheddar, A. Qammaz, and A. A. Argyros, "Towards force sensing from vision: Observing hand-object interactions to infer manipulation forces," in IEEE Conference on Computer Vision and Pattern Recognition, 2015, pp. 2810-2819.

[30] J. L. Elman, "Finding structure in time," Cognitive Science, vol. 14, no. 2, pp. 179-211, 1990.

[31] S. Hochreiter and J. Schmidhuber, "Long short-term memory," Neural computation, vol. 9, no. 8, pp. 1735-1780, 1997.

[32] T.-H. Pham, N. Kyriazis, A. A. Argyros, and A. Kheddar, "Hand-object contact force estimation from markerless visual tracking," IEEE Trans. on Pattern Analysis and Machine Intelligence, 2017, to appear.

[33] C. Fermüller, F. Wang, Y. Yang, K. Zampogiannis, Y. Zhang, F. Barranco, and M. Pfeiffer, "Prediction of manipulation actions," International Journal of Computer Vision, pp. 1-17, 2017.

[34] S. P. Boyd and L. Vandenberghe, Convex Optimization. New York, NY, USA: Cambridge University Press, 2004.

[35] S. Caron, Q.-C. Pham, and Y. Nakamura, "Stability of surface contacts for humanoid robots: Closed-form formulae of the contact wrench cone 
for rectangular support areas," in IEEE International Conference on Robotics and Automation, 2015, pp. 5107-5112.

[36] Y. Nakamura, K. Yamane, Y. Fujita, and I. Suzuki, "Somatosensory computation for man-machine interface from motion-capture data and musculoskeletal human model," IEEE Trans. on Robotics, vol. 21, no. 1, pp. 58-66, 2005.

[37] R. Dumas, L. Cheze, and J.-P. Verriest, "Adjustments to mcconville et al. and young et al. body segment inertial parameters," Journal of Biomechanics, vol. 40, no. 3, pp. 543-553, 2007.

[38] S. Caron, Q.-C. Pham, and Y. Nakamura, "Zmp support areas for multicontact mobility under frictional constraints," IEEE Trans. on Robotics, vol. 33, no. 1, pp. 67-80, 2016.

[39] S. Lengagne, Ö. Terlemez, S. Laturnus, T. Asfour, and R. Dillmann, "Retrieving contact points without environment knowledge," in IEEE RAS International Conference on Humanoid Robots, 2012, pp. 841-846.

[40] R. Collobert, K. Kavukcuoglu, and C. Farabet, "Torch7: A matlab-like environment for machine learning," in BigLearn, NIPS Workshop, 2011.

[41] N. Srivastava, G. Hinton, A. Krizhevsky, I. Sutskever, and R. Salakhutdinov, "Dropout: A simple way to prevent neural networks from overfitting," Journal of Machine Learning Research, vol. 15, no. 1, pp. 19291958, 2014.

[42] M. Andersen, J. Dahl, and L. Vandenberghe, "Cvxopt: A python package for convex optimization," abel.ee.ucla.edu/cvxopt, 2013.

[43] K. Bouyarmane and A. Kheddar, "Humanoid robot locomotion and manipulation step planning," Advanced Robotics, vol. 26, no. 10, pp. 1099-1126, 2012.

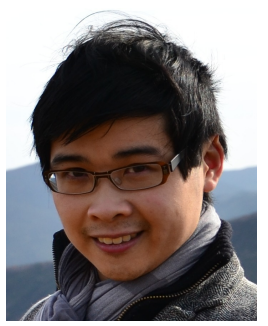

Tu-Hoa Pham is currently a postdoctoral researcher at IBM Research Tokyo. He received the Dipl.Ing. SupAéro degree from ISAE, the M.Sc. in Mathematics from Université Paul Sabatier (France, 2013) and the Ph.D. in robotics from Université de Montpellier (France, 2016), conducted between the CNRS-AIST Joint Robotics Laboratory, Japan, and CNRS-UM LIRMM, France. His research interests include robot vision and learning for monitoring of human activities and learning from demonstration.

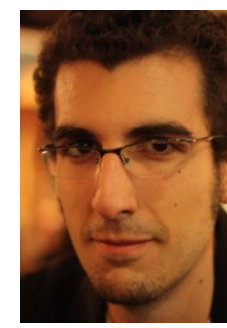

Stéphane Caron is a researcher in humanoid locomotion at CNRS-University of Montpellier LIRMM (France). An alumni of the École Normale Supérieure (ENS Paris), he stayed one year at the Technicolor Lab., Palo Alto (California), before joining the Nakamura Lab. to undergo his doctoral studies. He received the Ph.D. in Mechano-Informatics from the University of Tokyo (Japan) in 2016, with a thesis on multi-contact motion planning for humanoid robots. His research interests include contact interaction, numerical optimization and predictive control, all related to the broader field of humanoid locomotion.

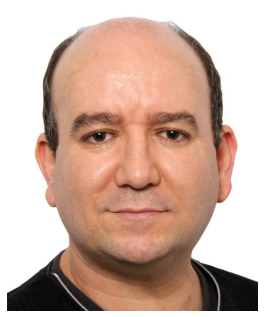

Abderrahmane Kheddar (M'04, SM'12) received the BS in Computer Science degree from the Institut National d'Informatique (ESI), Algiers, the MSc and $\mathrm{PhD}$ degree in robotics, both from the University of Pierre et Marie Curie, Paris. He is presently Directeur de Recherche at CNRS and the Director of the CNRS-AIST Joint Robotic Laboratory (JRL), UMI3218/RL, Tsukuba, Japan. He is also leading the Interactive Digital Humans (IDH) team at CNRS-University of Montpellier LIRMM, France. His research interests include haptics, humanoids and thought-based control using brain machine interfaces. He is a founding member of the IEEE/RAS chapter on haptics, the co-chair and founding member of the IEEE/RAS Technical committee on model-based optimization. $\mathrm{He}$ is a member of the steering committee of the IEEE Brain Initiative, Editor of the IEEE Transactions on Robotics and within the editorial board of some other robotics journals; he is a founding member of the IEEE Transactions on Haptics and served in its editorial board during three years (2007-2010). $\mathrm{He}$ is an IEEE senior member, titular member of the National Academy of Technology of France, and knight in the National Order of the Merit. 